\title{
Role of middle hepatic vein resection in standard right or left hepatectomy on post hepatectomy outcomes
}

\author{
Anisa NUTU*, Isaac JOHN, Bobby VM DASARI
}

Liver Unit, Queen Elizabeth Hospital Birmingham, Birmingham, United Kingdom

Introduction: Middle hepatic vein (MHV) is preserved in standard right or left hepatectomy in order to maintain the venous outflow of the remnant liver. Resection of MHV can cause venous parenchymal congestion, reflux of blood flow into portal vein, avoiding the regeneration of liver. The aim of this study was to evaluate if preservation or not of the MHV will influence post-surgical outcomes including post hepatectomy liver failure (PHLF) in standard right or left hepatectomy.

Methods: A retrospective analysis was carried out using a prospectively maintained database. A total of 144 patients underwent standard right or left hepatectomy (Brisbane 2000 nomenclature) between January 2015 and March 2019 were included. Anatomical remnant liver volumes were measured retrospectively using Hermes software.

Results: A right hepatectomy was performed in the $79 \%$ of the cases and a left one in the $21 \%$. MHV was resected in 13 patients (10\%) in addition to standard right or left hepatectomy. Median remnant liver volume in the MHV resected group was significantly more ( $p$ $=0.006)$. In the multivariable analysis, resection of the MHV did not influence in the occurrence of PHLF $(p=0.518)$. Similarly, there was no significant difference in the serum bilirubin, INR, ALT, creatinine levels on post op days 1, 3, 5, 10, and no significant difference in Grade IIIa or more complications, and 90-day mortality rates.

Conclusions: Resection of the MHV as a part of standard right or left hepatectomy in liver resection surgery did not have a negative impact on the post-operative outcomes in patients with adequate remnant liver volume. 